\title{
Post mortem radiology in children with congenital heart disease
}

\author{
G A RUSSELL, P J BERRY \\ From the Department of Paediatric Pathology, Bristol Royal Hospital for Sick Children, Bristol
}

SUMMARY Contrast radiology has valuable applications in routine necropsy practice. Contrast radiology was used to study the vascular anatomy in four normal fetuses, in five children with cardiovascular malformations, and in two hearts prepared by the perfusion fixation method. A contrast radiograph provides permanent documentation of anatomical associations before they are disrupted by dissection and permits a planned approach to the necropsy.

While these injection studies are seldom undertaken by pathologists because they are felt to be too difficult and time-consuming, contrast studies should form part of the post mortem investigation of children with congenital heart disease, even when angiography has been performed in life.

Post mortem radiology has a variety of applications in paediatric and adult necropsy investigations. A whole body $x$-ray is considered to be part of the routine examination of fetuses, ${ }^{1}$ perinatal, ${ }^{2}$ and sudden infant deaths. ${ }^{3}$ Contrast studies have been advocated in the demonstration of coronary artery disease after death ${ }^{4}$ and various malformations where in situ demonstration of the anomaly is required..$^{5}$ Many pathology departments now use self-contained $x$-ray machines to facilitate this work. We have used contrast injections in necropsy studies of cardiovascular malformations in children and have found them to be a valuable adjunct to other methods of examination.

\section{Material and methods}

Three groups of specimen were examined: (i) four anatomically normal fetuses; (ii) five children with congenital heart disease and associated vascular anomalies; (iii) and two perfusion fixed hearts with known malformations. A Faxitron machine (Model number 43856A, Hewlett Packard) was used with a base cabinet suitable for larger children. The first group allowed us to gain some experience in taking and interpreting contrast angiograms showing normal anatomy. The fetuses were in the range 24 to 40 weeks. Consent for necropsy had been obtained. A standard necklace incision permitted access to the blood vessels of the neck. The right jugular vein was dissected free to display the right common carotid artery. The cranial end of this vessel was ligated to prevent blood spillage

Accepted for publication 17 March 1988 and a small incision made in the exposed portion. An appropriate sized cannula was inserted and tied into the vessel. A plain anteroposterior $x$-ray was taken and developed on mammography film (3M-type $S$ ) as part of the standard necropsy procedure to ensure the correct machine settings. A film to source distance of $65 \mathrm{~cm}$ was used in the small cabinet. Five millilitres of date-expired contrast medium (Conray 420, May and Baker) were injected and the $x$-ray repeated in anteroposterior and lateral positions. Care was taken not to inject air with the contrast as this leads to unsightly and confusing bubbles in the radiograph. Contrast medium was washed out of the arterial tree using physiological saline. Water soluble contrast medium was selected because it was easily dispersed and did not interfere with subsequent dissection as barium gelatin might have. The right jugular vein was then cannulated and the process repeated for the venous system.

The second group studied were five children with clinical diagnoses of congenital heart disease in which anomalies of the major cardiac vessels were suspected (table). These children were older and larger than the first group but the method used was essentially the same. Routine blood cultures were taken before injection of contrast. The site of cannulation was selected to show the pathology to maximal advantage. Ten millitres of contrast medium were injected in these cases and adjustments to the machine settings were necessary because of the larger size of the children. Standard screened $x$-ray cassettes and the base cabinet of the Faxitron were used for this group with a film to source distance of $122 \mathrm{~cm}$. 
Table

\begin{tabular}{|c|c|c|c|c|}
\hline Case no & Age & $\operatorname{Sex}$ & Clinical diagnosis & Site \\
\hline 1 & 1 day & $\mathbf{M}$ & $\begin{array}{l}\text { Aortic valve atresia, single ventricle, } \\
\text { common atrioventricular canal }\end{array}$ & Right common carotid artery \\
\hline 2 & $\begin{array}{l}6 \text { months } \\
6 \text { months }\end{array}$ & $\begin{array}{l}\mathbf{F} \\
\mathbf{M}\end{array}$ & $\begin{array}{l}\text { Truncus arteriosus } \\
\text { Total anomalous pulmonary venous drainage to the } \\
\text { superior vena cava }\end{array}$ & $\begin{array}{l}\text { Right common carotid artery } \\
\text { Right internal jugular vein }\end{array}$ \\
\hline $\begin{array}{l}4 \\
5\end{array}$ & $\begin{array}{l}7 \text { days } \\
2 \text { days }\end{array}$ & $\begin{array}{l}\mathbf{M} \\
\mathbf{F}\end{array}$ & $\begin{array}{l}\text { Muscular ventricular septal defect, coarctation } \\
\text { Interrupted aortic arch }\end{array}$ & $\begin{array}{l}\text { Left common carotid artery } \\
\text { Left common carotid artery }\end{array}$ \\
\hline
\end{tabular}
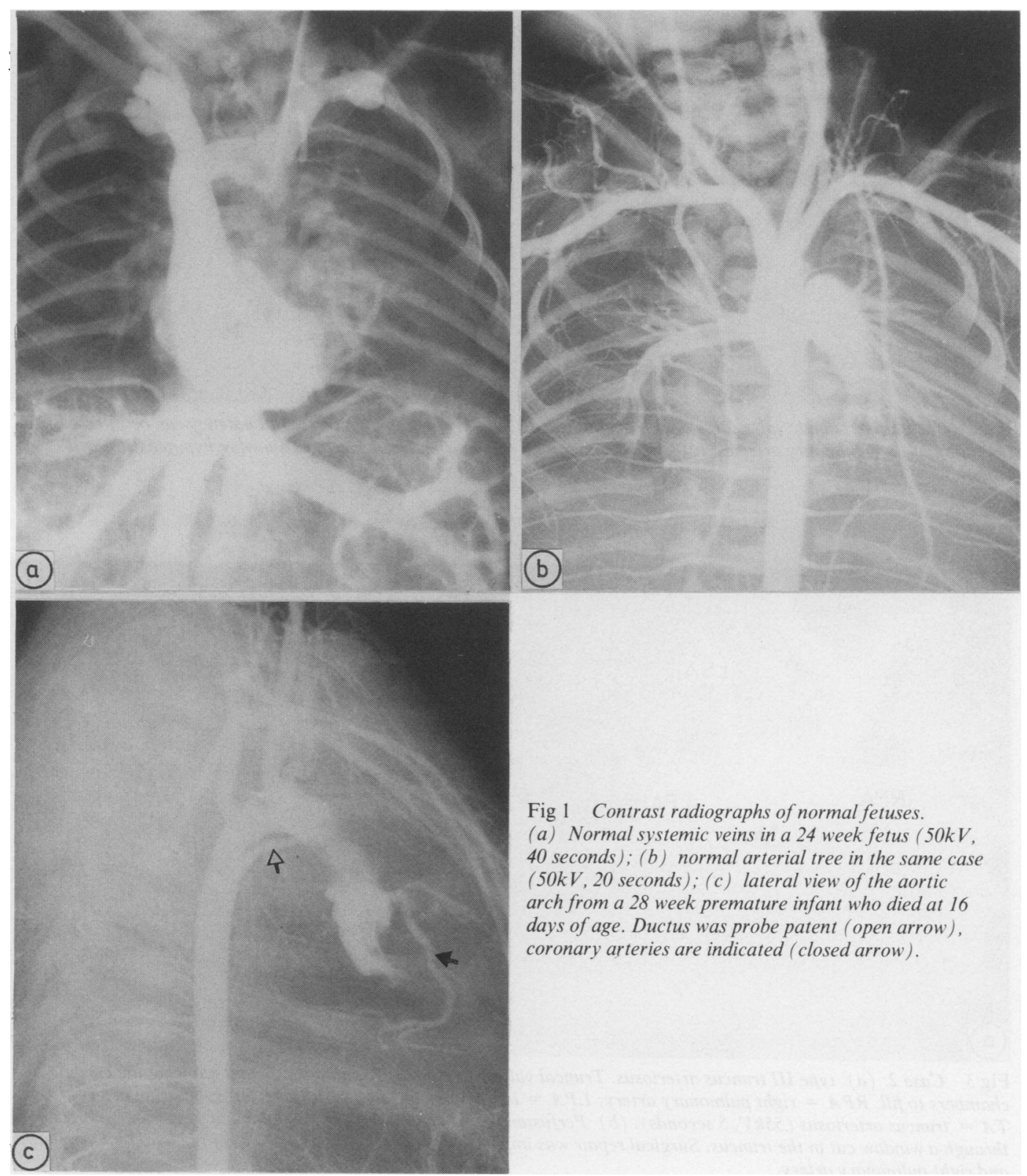

Fig 1 Contrast radiographs of normal fetuses.

(a) Normal systemic veins in a 24 week fetus $(50 \mathrm{kV}$,

40 seconds); (b) normal arterial tree in the same case

(50k V, 20 seconds); (c) lateral view of the aortic arch from a 28 week premature infant who died at 16 days of age. Ductus was probe patent (open arrow), coronary arteries are indicated (closed arrow). 


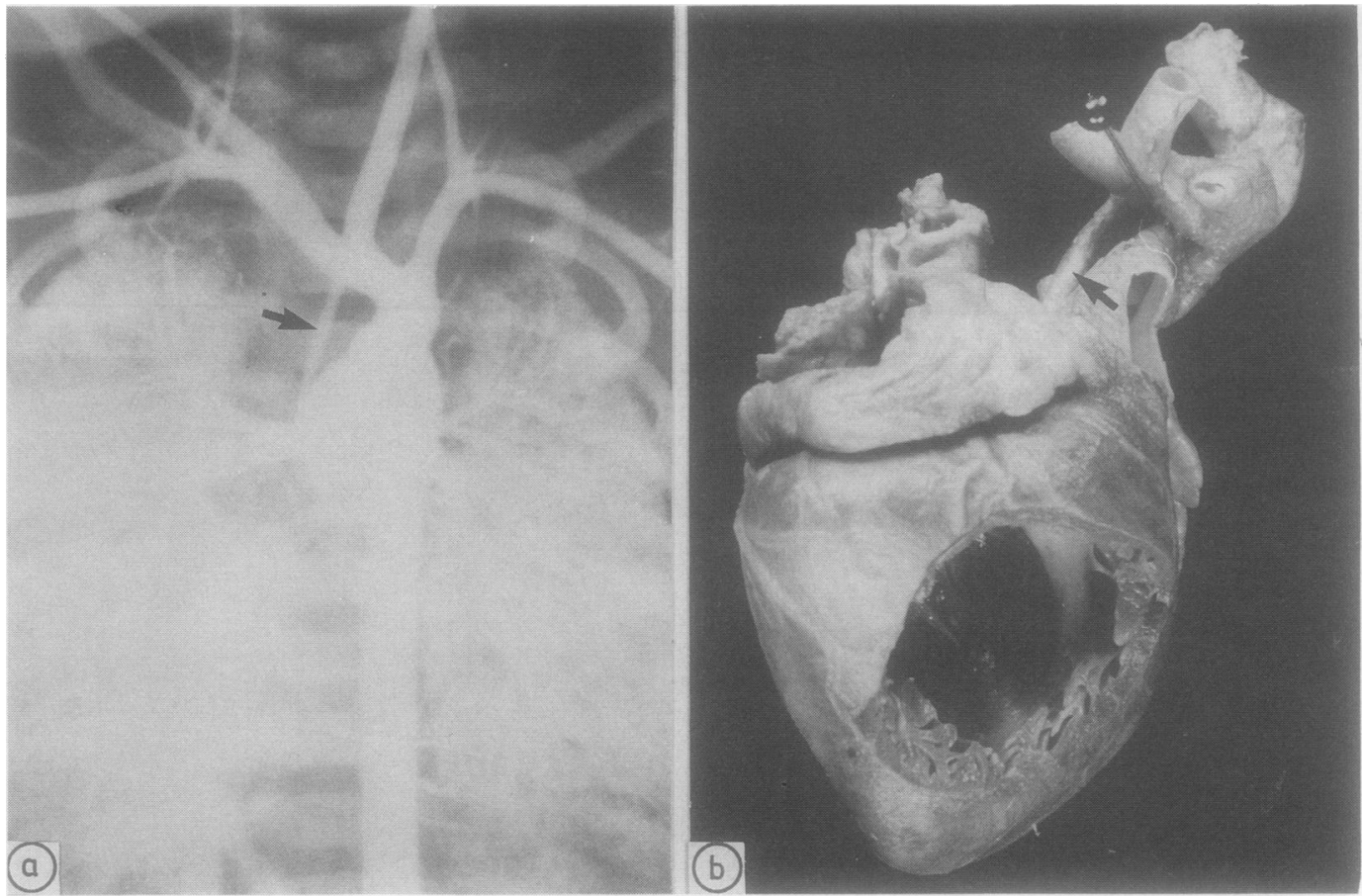

Fig 2 Case 1: (a) aortic valve atresia with hypoplastic ascending aorta (arrow). Ductal patency can be inferred from the filling of the pulmonary arteries. (50kV, 4 seconds). (b) Perfusion fixed specimen showing hypoplastic aorta (arrow).

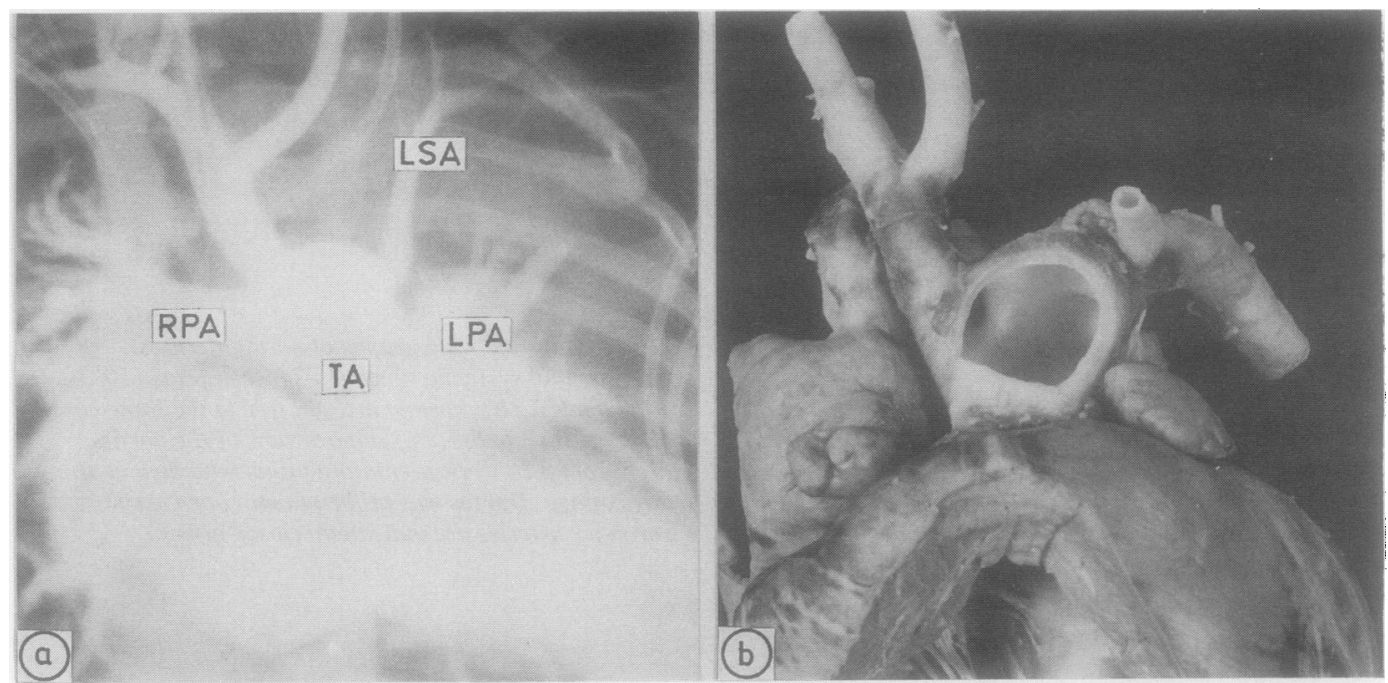

Fig 3 Case 2: (a) type III truncus arteriosus. Truncal valve was dysplastic and incompetent allowing the cardiac chambers to fill. $R P A=$ right pulmonary artery; $L P A=$ left pulmonary artery; $L S A=$ left subclavian artery; $T A=$ truncus arteriosus $(55 \mathrm{kV}, 5$ seconds). (b) Perfusion fixed heart. Origin of the pulmonary arteries is visible through a window cut in the truncus. Surgical repair was impossible because of the proximity of the innominate artery and right pulmonary artery. 


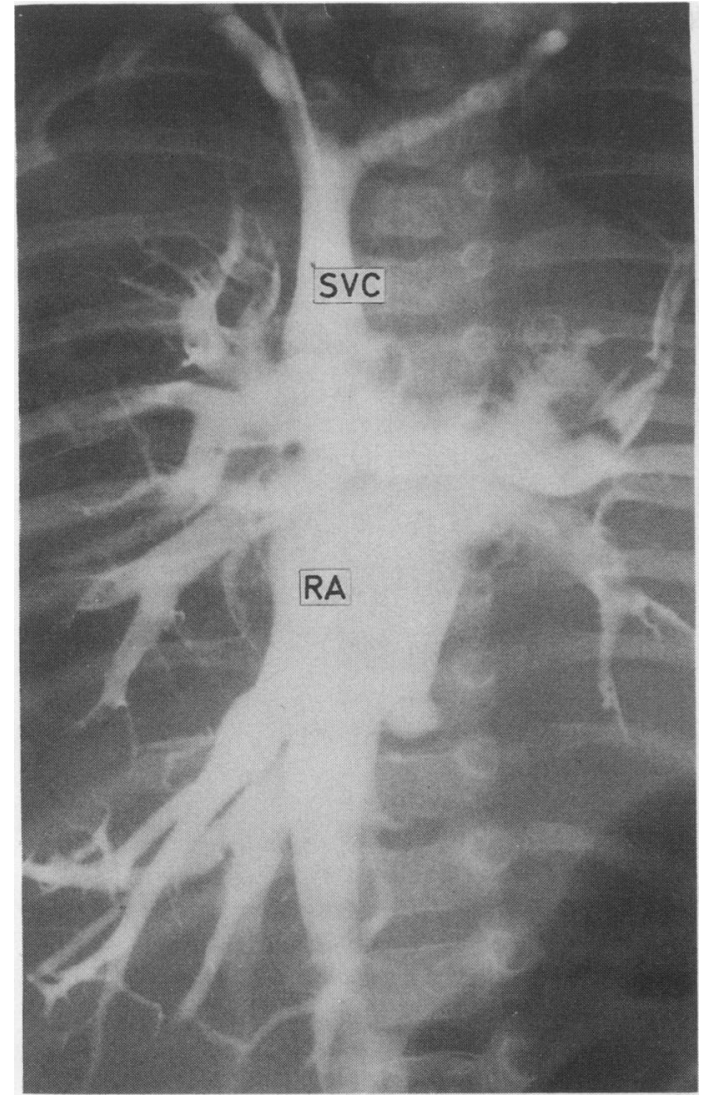

Fig 4 Case 3: total anomalous pulmonary venous drainage. Pulmonary veins drain into the superior vena cava $(S V C)$ just above its confluence with the right atrium ( $R A)$ ( $55 k V, 5$ seconds).

The third group were two hearts prepared by the perfusion fixation method described by Thomas and Davies. ${ }^{6}$ The first heart was from a child of 18 months with a single ventricle and atresia of both the mitral and pulmonary valves. Contrast injections were made to assess the patency of bilateral Blalock shunts, one of which was thought to be obstructed. Contrast $(5 \mathrm{ml})$ was injected into the left pulmonary artery with other major arteries tied off and a clip across the aorta. The second case was the heart of a 1 day old child with a diagnosis of a cardiomyopathy made by antenatal echocardiography. Contrast medium was injected into the right common carotid artery of the fixed specimen. Air was introduced into the left ventricle to obtain a "double-contrast" type of picture.

\section{Results}

The work on the normal fetuses showed the ease with which angiograms can be obtained on necropsy specimens (fig 1). Clots did not seem to be an appreciable problem in large vessels. The lateral view proved useful for examination of the aortic arch and ductus (fig lc). Contrast medium did not fill the heart, although coronary filling did give some idea of its location. Other viscera could be delineated by their vascular anatomy.

In all five cases of congenital heart disease the contrast angiogram provided clear demonstration of the vascular anatomy (figs $2-6$ ). In case 2 the $x$-ray taken at necropsy provided particularly valuable clinical correlation as the limited catheter studies performed in life had been difficult to interpret. In case 5 the angiogram indicated the presence of an aortopulmonary window before dissection was started. Again, no direct demonstration of associated intracardiac malformations was obtained from these studies.

Contrast $x$-rays were also of value in the two perfused hearts examined. In the first case both shunts were widely patent, refuting the clinical and echocardiographic diagnoses. Subsequent dissection showed closure of an atrial septal defect as the cause of this child's deterioration (fig 7a). The $x$-ray demonstration of shunt patency allowed us to preserve the dissected specimen intact for wax impregnation ${ }^{7}$ and use at clinical meetings (fig $7 \mathrm{~b}$ ). It also gave a more accurate estimate of the degree of patency than crude probing would have done. In the second case the $x$-ray of the heart allowed a detailed assessment of the cardiac anomalies to be made before dissection. It also allowed us to select the most appropriate plane of section to display the anomalies (fig 8). The contrast outlined a narrow ascending aorta and tracked into the left ventricle, indicating that the aortic valve was stenotic rather than atretic. The air in the left ventricle showed the abnormal globular shape of the chamber with high papillary muscle insertion and defined the dome-shaped stenotic aortic valve. Subendocardial calcification was a useful clue as to the presence of areas of myocardial necrosis.

\section{Discussion}

Plain $x$-rays taken at necropsy only delineate the vascular anatomy if there is calcification of the wall or calcified thrombus within the lumen (fig 9). Contrast techniques are usually needed if vessels are to be shown. Our study shows that contrast radiology is cheap and easy to perform. $X$-ray examination should not be considered as an alternative to a standard necropsy. In cases where consent for necropsy is refused, however, bereaved relatives may give permission for angiograms to be performed through a limited and easily concealed incision. In some postoperative cases angiograms may be performed 

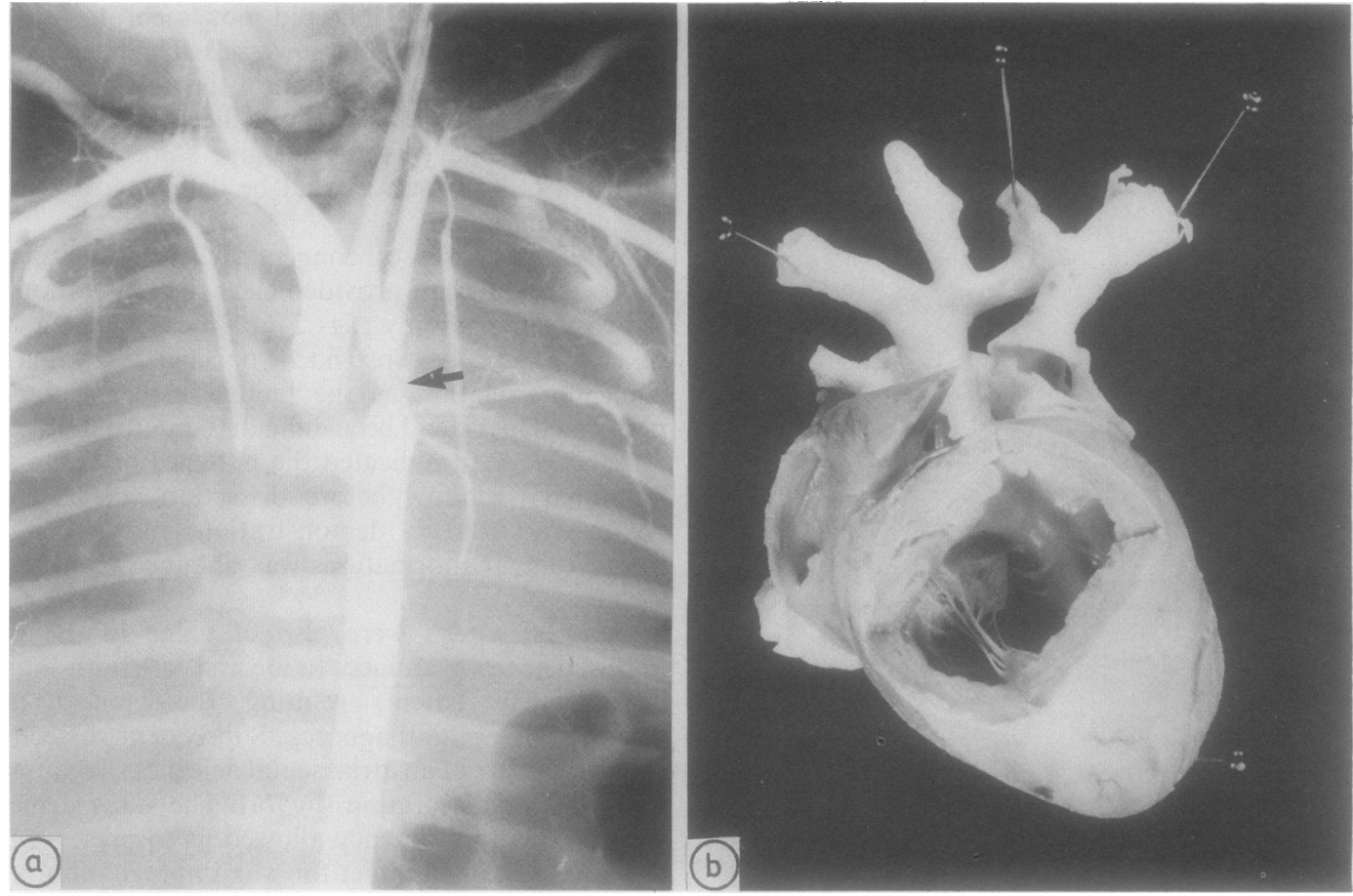

Fig 5 Case 4: (a) preductal coarctation of the aorta (arrow). Ductus was patent but had not filled (55kV, 5 seconds). (b) Coarctation is not very obvious on the necropsy specimen. Effective $x$-ray demonstration of the lesion allowed the whole arch to be processed for optimal histology without opening the vessels.
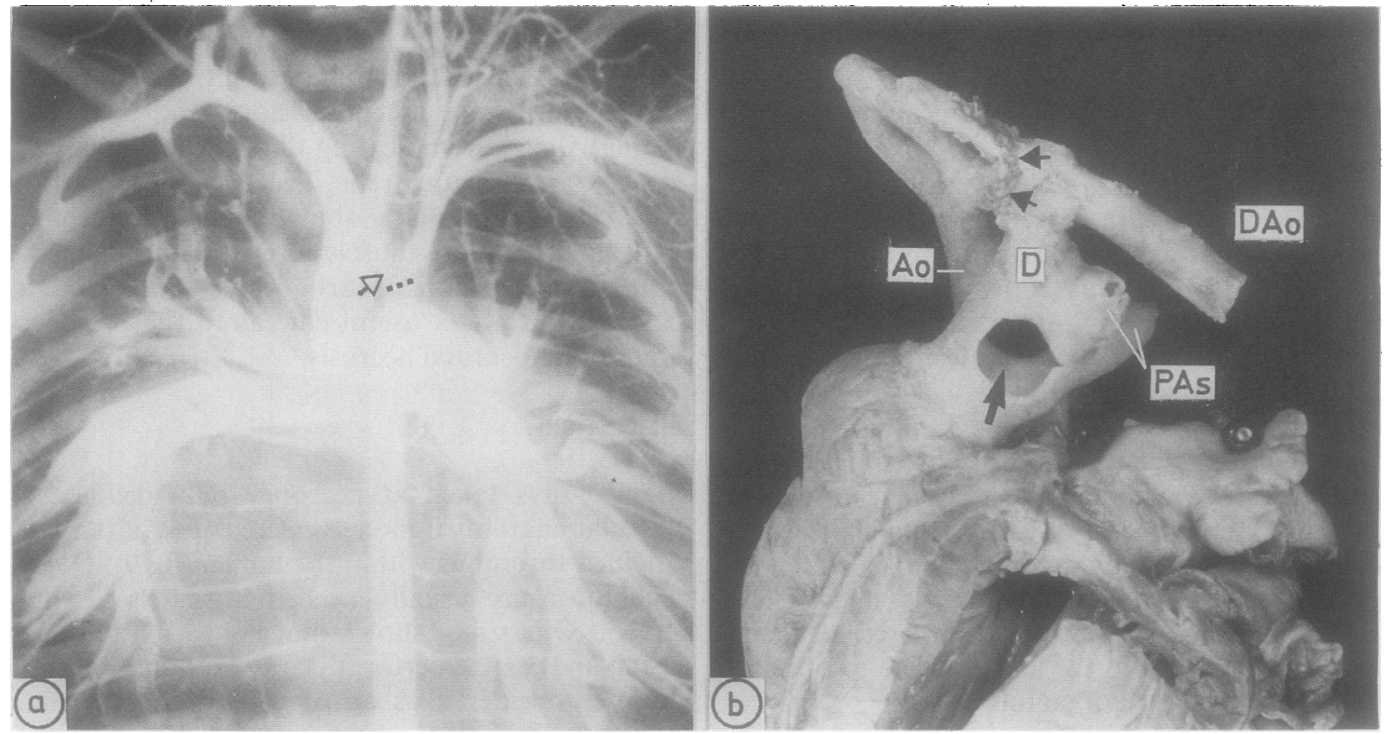

Fig 6 Case 5: (a) surgical repair of a "type a" aortic arch atresia (dotted line represents the anastamosis just distal to the left subclavian artery). X-ray picture shows severe residual narrowing of the aortic arch (arrow) and possible collateral vessels in the left neck. Ductus was tied at operation, yet there is filling of the pulmonary arteries, implying the presence of residual shunt ( $55 \mathrm{kV}, 5$ seconds). (b) Aortopulmonary window is visible (arrow) accounting for the filling of the pulmonary arteries $(P A s) . A o=$ aorta $; D A o=$ descending aorta; $D=$ ligated ductus. 


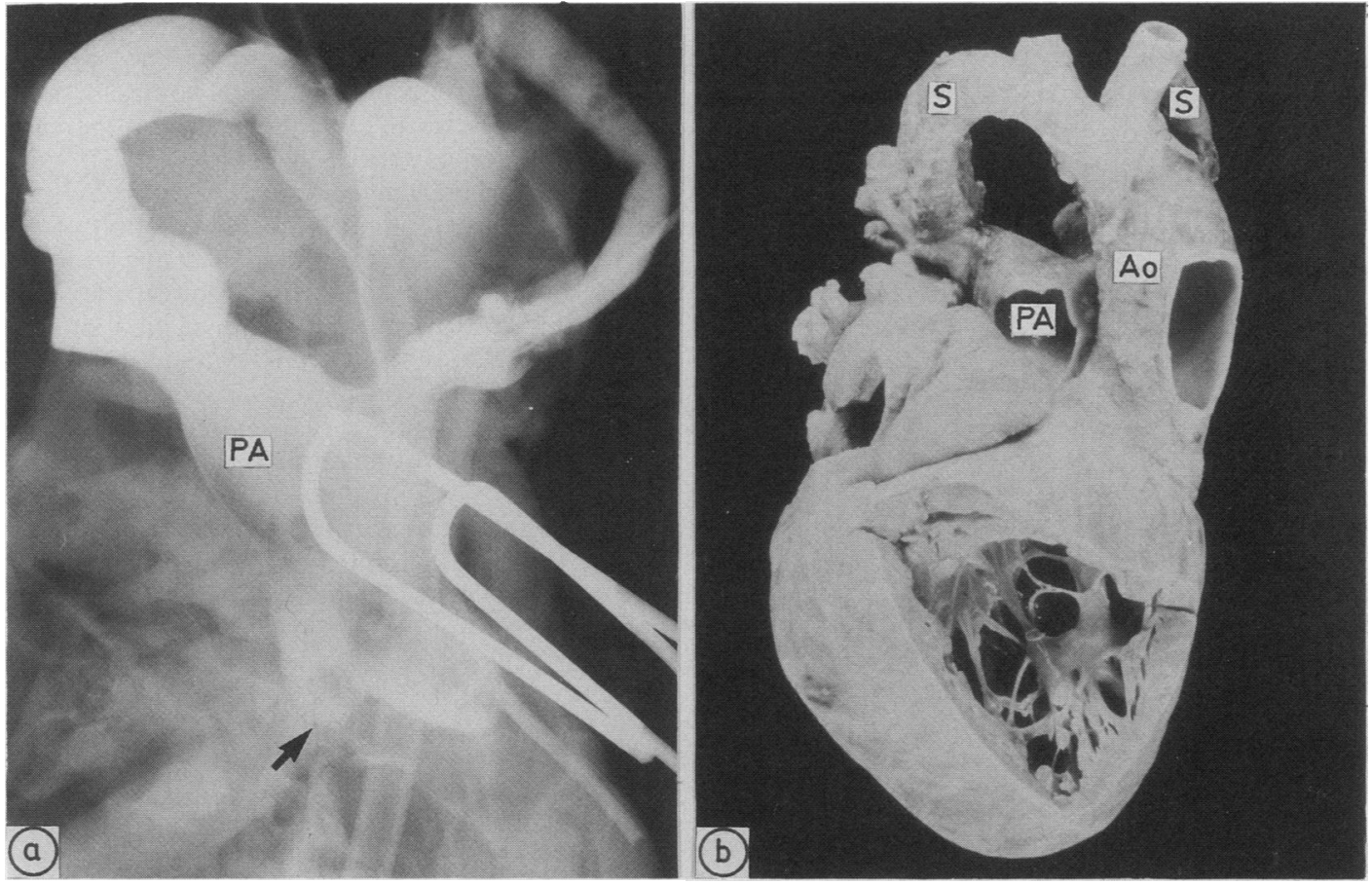

Fig 7 a Contrast study of the heart from a child with known mitral and pulmonary atresia. Suspected shunt obstruction was diagnosed but $x$-ray picture shows patency of both shunts. Aorta has been closed by a clip to prevent spillage. Note hypoplastic pulmonary artery (PA) with atretic valve (arrow) ( $50 \mathrm{kV}, 30$ seconds).

Fig $7 \mathrm{~b}$ Perfusion fixed specimen showing transposed aorta $(\mathrm{Ao})$ and pulmonary artery $(\mathrm{PA}) ;(\mathrm{S})=$ bilateral Blalock shunts. Spontaneous closure of an atrial septal defect had been the actual cause of the clinical deterioration.
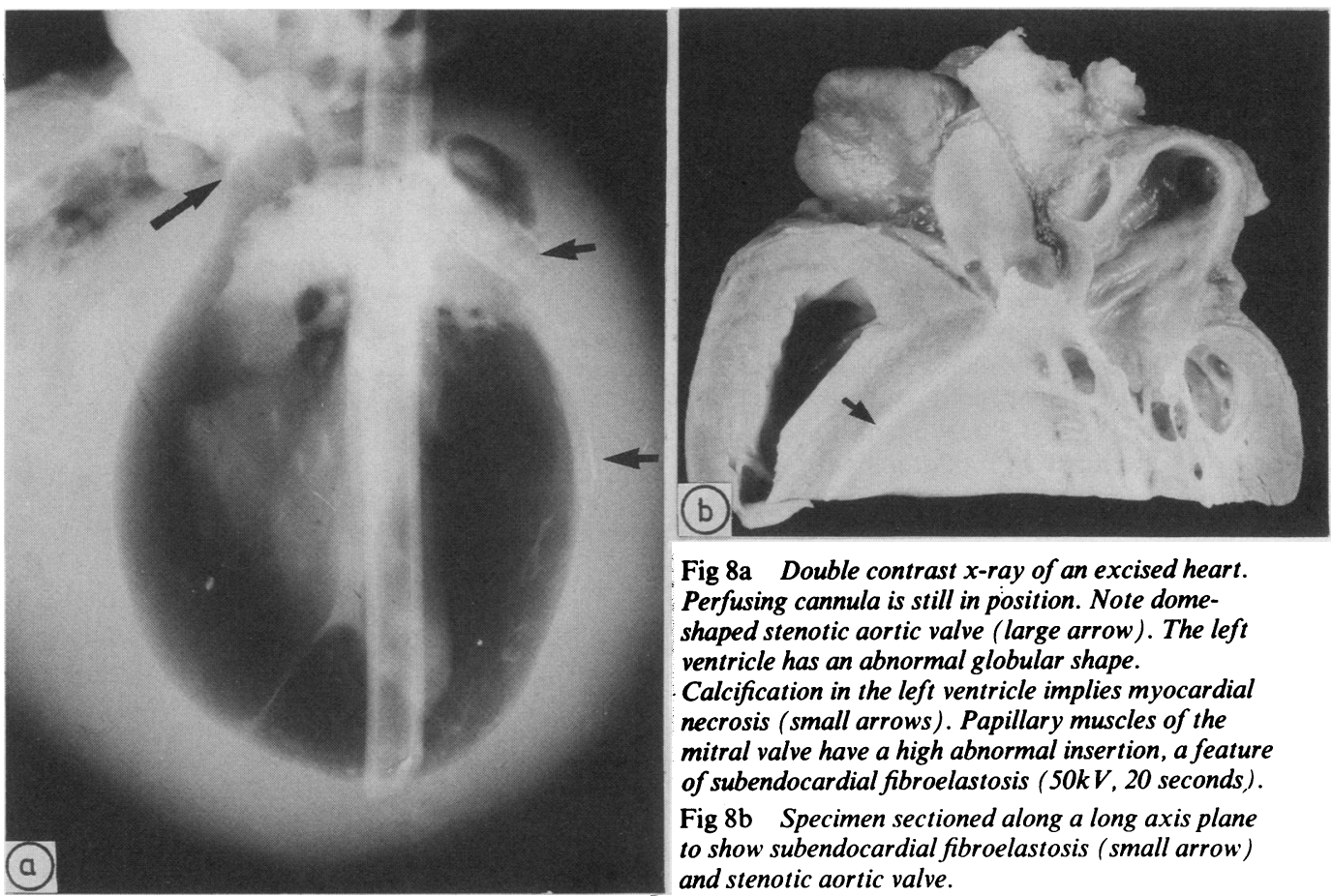

Fig $8 \mathrm{a}$ Double contrast $x$-ray of an excised heart. Perfusing cannula is still in position. Note domeshaped stenotic aortic valve (large arrow). The left ventricle has an abnormal globular shape.

Calcification in the left ventricle implies myocardial necrosis (small arrows). Papillary muscles of the mitral valve have a high abnormal insertion, a feature of subendocardial fibroelastosis ( $50 \mathrm{kV}, 20$ seconds).

Fig 8b Specimen sectioned along a long axis plane to show subendocardial fibroelastosis (small arrow) and stenotic aortic valve. 


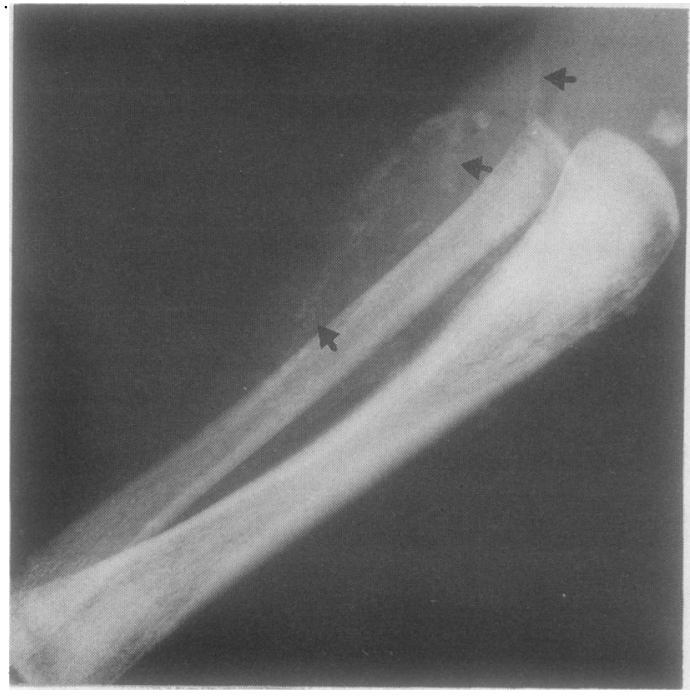

Fig 9 Calcified thrombus in the calf veins of a 6 week old child due to parenteral alimentation ( $50 \mathrm{kV}, 30$ seconds).

through an in-dwelling vascular cannula, although larger volumes of contrast medium may be required. The radiographs obtained give a vivid and permanent record of precise anatomical relationships, which may be destroyed or distorted by dissection. Thus they provide valuable correlation with catheter and echocardiographic studies. They are of high quality because of the relatively large volumes of contrast injected. The $x$-ray may alert the pathologist to a lesion and allow him or her to modify the technique to best show the pathology. The calibre of vessels is easily measured from the angiogram and gives a more accurate figure than might be obtained from probing the vascular lumen. Small collateral vessels may be easily overlooked at necropsy but will be well displayed by these techniques.

Post mortem contrast radiology can still be used once the heart has been removed. Information about the patency and calibre of valves, vessels, and shunts is easy to obtain and may avoid the necessity for destructive dissection of the specimen. Calcification of myocardial infarcts in children with cardiac malformations is not uncommon. The extent of calcification may be visible on $x$-rays of the whole specimen or slices of the ventricles. Simple doublecontrast effects can be used to outline valves and chambers. More complex double-contrast methods have been described, ${ }^{8}$ but these require the cardiac chambers to be opened before study. Of course, the postmortem angiographic study provides no functional information about the direction of blood flow or pressures. Interpretation must always be in the light of subsequent dissection. Nevertheless, these simple methods permit optimal dissection and closer clinical correlation from necropsy material. We agree with Foote et al ${ }^{9}$ who concluded that the use of contrast media after death can assist in the delineation of complex cardiovascular malformations.

We thank the Department of Radiology, Bristol Royal Hospital for Sick Children for materials and advice. We also thank our clinical colleagues in the departments of cardiology and cardiac surgery for their assistance.

\section{References}

1 Barson AJ. The perinatal postmortem. In: Barson AJ, ed. Fetal and neonatal pathology. London: Royal College of Pathologists, 1982:153.

2 Keeling JW. Postmortem examination of the fetus. In: Rodeck $\mathrm{CH}$, Nicolaides KH, eds. Prenatal diagnosis. London: Royal College of Obstetricians and Gynaecologists, 1984:351.

3 Wigglesworth JS, Keeling JW, Rushton DI, Berry PJ. Pathological investigations in cases of sudden infant death. J Clin Pathol 1987;40:1481-3.

4 Coghill SB, Nicoll SM, McKimmie A, Houston I, Mathews BM. Revitalising postmortem coronary angiography. J Clin Pathol 1983;36:1406-9.

5 Keeling JW. The perinatal autopsy. In: Keeling JW, ed. Fetal and neonatal pathology. London: Springer Verlag, 1987:10.

6 Thomas AC, Davies MJ. The demonstration of cardiac pathology using perfusion fixation. Histopathology 1985;9:5-19.

7 Russell GA, Berry PJ. Approaches to the demonstration of congenital heart disease. J Clin Pathol 1986;39:503-7.

8 Wright LS, Jaffe R, Lewis D, Figley MM. A method for doublecontrast stereoscopic study of the internal anatomy of the heart. Radiology 1964;83:1003-7.

9 Foote GA, Wilson AJ, Stewart JH. Perinatal postmortem radiography-experience of 2500 cases. $\mathrm{Br} J$ Radiol 1978; 51:351-6.

Requests for reprints to: Dr G A Russell, Department of Histopathology, Southmead Hospital, Westbury-on-Trym, Bristol BS10 5NB, England. 